\title{
Outer Membrane Vesicles of Gram-Negative Bacteria: An Outlook on Biogenesis
}

\section{OPEN ACCESS}

Edited by:

Daniela De Biase,

Sapienza University of Rome, Italy

Reviewed by:

Paola Sperandeo,

University of Milan, Italy

Meta J. Kuehn,

Duke University, United States Jonathan Lynch, University of California, Los Angeles, United States

${ }^{*}$ Correspondence: Araceli Contreras-Rodríguez aracelicontreras21@gmail.com;

acontrerasr@ipn.mx

Eric Daniel Avila-Calderón mosi6286@hotmail.com

Specialty section:

This article was submitted to Microbial Physiology and Metabolism,

a section of the journal

Frontiers in Microbiology

Received: 30 April 2020 Accepted: 04 February 2021

Published: 04 March 2021

Citation:

Avila-Calderón ED, Ruiz-Palma MdS, Aguilera-Arreola MG, Velázquez-Guadarrama N, Ruiz EA,

Gomez-Lunar Z, Witonsky S and Contreras-Rodríguez A (2021) Outer

Membrane Vesicles of Gram-Negative Bacteria: An Outlook

on Biogenesis.

Front. Microbiol. 12:557902.

doi: 10.3389/fmicb.2021.557902

\author{
Eric Daniel Avila-Calderón ${ }^{1,2 *}$, María del Socorro Ruiz-Palma ${ }^{1,3}$, Ma. Guadalupe \\ Aguilera-Arreola ${ }^{1}$, Norma Velázquez-Guadarrama ${ }^{4}$, Enrico A. Ruiz', \\ Zulema Gomez-Lunar ${ }^{1}$, Sharon Witonsky ${ }^{6,7}$ and Araceli Contreras-Rodríguez ${ }^{1 *}$
}

'Departamento de Microbiología, Escuela Nacional de Ciencias Biológicas, Instituto Politécnico Nacional, México City, Mexico, ${ }^{2}$ Departamento de Biología Celular, Centro de Investigación y de Estudios Avanzados, Instituto Politécnico Nacional, CINVESTAV-IPN, México City, Mexico, ${ }^{3}$ División Químico Biológicas, Universidad Tecnológica de Tecámac, Tecámac, Mexico, ${ }^{4}$ Unidad de Investigación en enfermedades infecciosas, Hospital Infantil de México Federico Gómez, Ciudad de México, Mexico, ${ }^{5}$ Departamento de Zoología, Escuela Nacional de Ciencias Biológicas, Instituto Politécnico Nacional, México City, Mexico, ${ }^{6}$ Center for One Health Research, Virginia-Maryland College of Veterinary Medicine, Virginia Tech, Blacksburg, VA, United States, 'Large Animal Clinical Sciences, Virginia-Maryland College of Veterinary Medicine, Virginia Tech, Blacksburg, VA, United States

Outer membrane vesicles (OMVs) from Gram-negative bacteria were first described more than 50 years ago. However, the molecular mechanisms involved in biogenesis began to be studied only in the last few decades. Presently, the biogenesis and molecular mechanisms for their release are not completely known. This review covers the most recent information on cellular components involved in OMV biogenesis, such as lipoproteins and outer membrane proteins, lipopolysaccharide, phospholipids, quorum-sensing molecules, and flagella.

Keywords: outer membrane vesicles, bacterial vesicles, extracellular vesicles, OMVs biogenesis, phospholipids, LPS, PQS, flagellin

\section{INTRODUCTION}

Outer membrane vesicles (OMVs) are nanostructures released by pathogenic and non-pathogenic Gram-negative bacteria in vivo and in vitro. OMVs range in size from 20 to $300 \mathrm{~nm}$ and are released during bacterial growth (Jan, 2017). These vesicles are formed from the bacterial outer membrane; thus, they contain phospholipids, lipopolysaccharide (LPS), outer membrane proteins (OMPs), and periplasmic proteins trapped during membrane budding (Jan, 2017). Because of their composition, these vesicles have been linked to many physiological processes, such as protein transport, nutrient acquisition, cell-intercellular communication, antibacterial activity, toxin delivery, and host-immune response modulation (Avila-Calderón et al., 2015).

Outer membrane vesicle generation begins with outer membrane bulging and ends with the release of vesicles into the external surroundings. The molecular mechanism for OMV production is still unclear. However, genetic and biochemical evidence has revealed some clues towards a better understanding of this complex process (Pathirana and Kaparakis-Liaskos, 2016).

The present review discusses new findings concerning OMV biogenesis as well as some important molecular determinants involved in vesicle formation, including lipoproteins, LPS, phospholipids, quorum-sensing molecules, and flagella. 


\section{Models for OMV Biogenesis}

The first observation of bacterial vesicles dates back to the 1960s, but only in recent decades has there been a significant increase in reports on the biogenesis, physiological roles, and applications of OMVs. Some of the first reports were performed using Escherichia coli strains, and researchers observed that the lysine-requiring mutant E. coli 12,408 produced "globules" surrounded by membranes with no evidence of cell lysis (Knox et al., 1966). Moreover, E. coli JC411 released vesicles containing lipids and proteins (Hoekstra et al., 1976). Other experiments using E. coli W3110 showed that membrane vesicles were released from the cell surface after heating the cells at $55^{\circ} \mathrm{C}$. These vesicles contained lipid, LPS, and protein compositions similar to those of the outer membrane from whole cells (Katsui et al., 1982). After these findings were reported, the number of published studies concerning OMVs has increased (Gamazo and Moriyón, 1987; Grenier and Bélanger, 1991; Kadurugamuwa and Beveridge, 1995).

Three models propose a role for lipoproteins, LPS, and peptidoglycan in the biogenesis of OMVs. Recently, new reports contributed to the knowledge of OMV production, some of which agree with the early models proposed, whereas others shed light on new molecules involved in vesiculation. In this section, the three early models are described initially; in the subsequent section, new insights into the molecular determinants involved in OMVs are described.

The first model explains the formation of OMVs due to the low number of lipoproteins attached to the peptidoglycan layer. The low number of lipoprotein linkages leads to outer membrane bulging, affecting vesicle production. In 1976, Hoekstra et al. determined the number of lipoproteins found in the outer membrane of E. coli as well as those contained in the vesicles. These authors reported fewer lipoproteins in the vesicles than in the outer membrane. Based on these results, the authors proposed that vesicles are released from outer membrane sites with few lipoprotein linkages (Hoekstra et al., 1976; Mashburn-Warren and Whiteley, 2006). The second model was based on the presence of peptidoglycan residues with autolysins in the OMVs. This model proposes that during synthesis of the peptidoglycan layer, sites exist where the concentration of peptidoglycan is higher, causing protrusions in the outer membrane and indicating the beginning of vesicle formation. An important finding that supported this model was the presence of muramic acid, a known peptidoglycan layer precursor, in OMVs purified from Porphyromonas gingivalis (Zhou et al., 1998). Furthermore, mutation of an autolysin involved in peptidoglycan replacement increased the synthesis of OMVs (Hayashi et al., 2002). These findings suggest that the accumulation of peptidoglycan residues increases outer membrane bulging, triggering the release of OMVs (Zhou et al., 1998; Hayashi et al., 2002). The third and last model involves the electric charge of LPS in OMV formation. Pseudomonas aeruginosa produces two types of LPS: negatively charged LPS and neutrally charged LPS. OMVs released by $P$. aeruginosa cultured under oxidative stress conditions primarily comprise negatively charged LPS. Therefore, an increase in negatively charged LPS within the cell envelope was proposed to favor the release of OMVs because of the repulsion caused by negative charges in the outer membrane (Kadurugamuwa and Beveridge, 1995; Sabra et al., 2003).

These models highlighted the importance of lipoproteins, LPS, and peptidoglycan during OMV formation. However, it remains unknown whether these mechanisms act in tandem. Some of the most important and widely accepted mechanisms for $\mathrm{OMV}$ formation are outlined in the following sections.

\section{Some Lipoproteins and the Outer Membrane Protein OmpA Are Involved in OMV Biogenesis}

The envelope of Gram-negative bacteria is made up of the inner and outer membranes, which are separated by the periplasmic space. Peptidoglycan is bound to proteins in the outer and inner membranes through covalent and noncovalent bonds (Silhavy et al., 2010). Peptidoglycan is composed of linear glycans (g $\beta$-1,4-connected N-acetylglucosamine and $\mathrm{N}$-acetylmuramic acid) that are cross-linked by short peptides (Pazos and Peters, 2019). Braun's lipoprotein, commonly referred to as Lpp, is the major lipoprotein in E. coli and is the only lipoprotein covalently that is linked to the peptidoglycan layer and plays a unique role in the envelope architecture. The $\mathrm{N}$-terminal domain of Lpp is acylated and inserted into to the outer membrane, while the C-terminal domain is covalently linked to the peptidoglycan layer (Lee and Inouye, 1974). OmpA in $E$. coli is a $\beta$-barrel, and its C-terminal domain interacts with peptidoglycan through a 20-aa residue linker region (Smith et al., 2007). In 2017, Samsudin et al. have shown that interactions among OmpA, peptidoglycan, and Lpp are essential in maintaining the integrity of the cellular envelopes. The research team showed that Lpp aids in the interaction of monomeric OmpA with the peptidoglycan layer. In the absence of Lpp, the C-terminal domain of OmpA binds to the outer membrane. Additionally, an OmpA homodimer can easily interact with the peptidoglycan layer in the absence of Lpp (Samsudin et al., 2017). These lipoproteins and outer membrane proteins are associated with outer membrane stability.

Initially, the production of "blebs" (OMVs) was considered an alteration or instability of the outer membrane rather than a physiological phenomenon. For example, in 1969, Koike et al. observed bleb formation on the surface of E. coli after treatment with polymyxin B. However, the same authors observed that polymyxin $B$ induced outer membrane projections rather than blebs in $P$. aeruginosa. These projections diminished as the concentration of the antibiotic was decreased. Thus, blebs and outer membrane projections were considered to be affected by the action of the antibiotic on the outer membrane (Koike et al., 1969). Subsequently, the release of OMVs was observed in more bacterial species, and vesicles were found not to be the product of cell lysis (Zhou et al., 1998). The exclusion of some periplasmic and outer membrane components and enrichment of other components in vesicles led to the consideration of the existence of a specific mechanism to select molecules carried on OMVs (Kadurugamuwa and Beveridge, 1995; Horstman and Kuehn 2000; Kato et al., 2002; Bonnington and Kuehn, 2014). Based on this background, "OMV release" is the product 
of a physiological phenomenon but not the product of cell lysis or membrane instability.

In 1976 and 1978, the first molecular evidence regarding OMV biogenesis was revealed by Weigand et al. whose results were obtained using Salmonella enterica serovar Typhimurium (hereafter referred to as Salmonella typhimurium) and Suzuki et al. whose work focused on E. coli. Their works showed that the E. coli lpo mutant and S. typhimurium $l k y D$ mutant lacking the murein lipoprotein (the product of these genes was later designated Braun's lipoprotein or Lpp) released "blebs" from the outer membrane. Although Suzuki et al. (1978) observed the production of blebs from Salmonella cells, Weigand et al. (1976) reported bleb production at the septal region in E. coli. Bernadac et al. (1998) studied two mutated E. coli strains, the lpp and ompA mutants, both of which exhibited a hypervesiculation phenotype. Similarly, Lpp was also associated with OMV production in bacterial species such as Yersinia pestis (Eddy et al., 2014). The hypervesiculation phenotype was also observed in Acinetobacter baumannii and Vibrio cholerae ompA (Moon et al., 2012; Valeru et al., 2014). A. baumannii, V. cholerae, and other Gram-negative bacteria with a mutation in the ompA gene could not establish bonds between the peptidoglycan and the outer membrane, leading to OMV overproduction (Figure 1; Jin et al., 2011; Moon et al., 2012). These results demonstrate that lipoproteins and the outer membrane proteins involved in membrane stability are linked to OMV biogenesis.

The role of Lpp and OmpA in the formation of OMVs has also been analyzed. However, some discrepancies were observed in the results obtained by different research groups. In 2009, Deatherage et al. analyzed the effect on OMV formation in S. typhimurium LT2 caused by specific mutations in the $l p p$ and ompA genes. Further experiments on the S. typhimurium LT2 tolA, tolB, and pal mutants suggested that they could not form outer-membrane-peptidoglycan-inner membrane linkages, and vesicle formation was observed around the cell body. Weigand et al. (1976) reported OMV formation in the septa of the S. typhimurium lpp mutant. However, Deatherage et al. (2009) observed vesicle formation in the cell bodies of different S. typhimurium lpp gene mutants. Differences observed in both studies can be attributed to $S$. typhimurium harboring two copies of the lipoprotein gene that are separated by 82 bp $(l p p A$ and $l p p B)$. Deatherage et al. (2009) mutated both $l p p$ genes, while Weigand mutated only one of them. These findings showed two possible hypotheses: (i) the bonds between the outer membrane and peptidoglycan layer dissociate, leading to OMV release into the periphery of the cell body (Deatherage et al., 2009); and (ii) during bacterial division, the bonds between the peptidoglycan layer and inner membrane decrease within the division septum, affecting the number of linkages among the cytoplasmic membrane, cell wall, and outer membrane, and leading to membrane protrusion and vesicle release (Deatherage et al., 2009). OMVs are released from either the septa or cell body likely because of specific interactions among Lpp, OmpA, and peptidoglycan. OmpA, as a monomer or homodimer, interacts with peptidoglycan and the outer membrane in the presence or absence of Lpp. Most likely, the presence

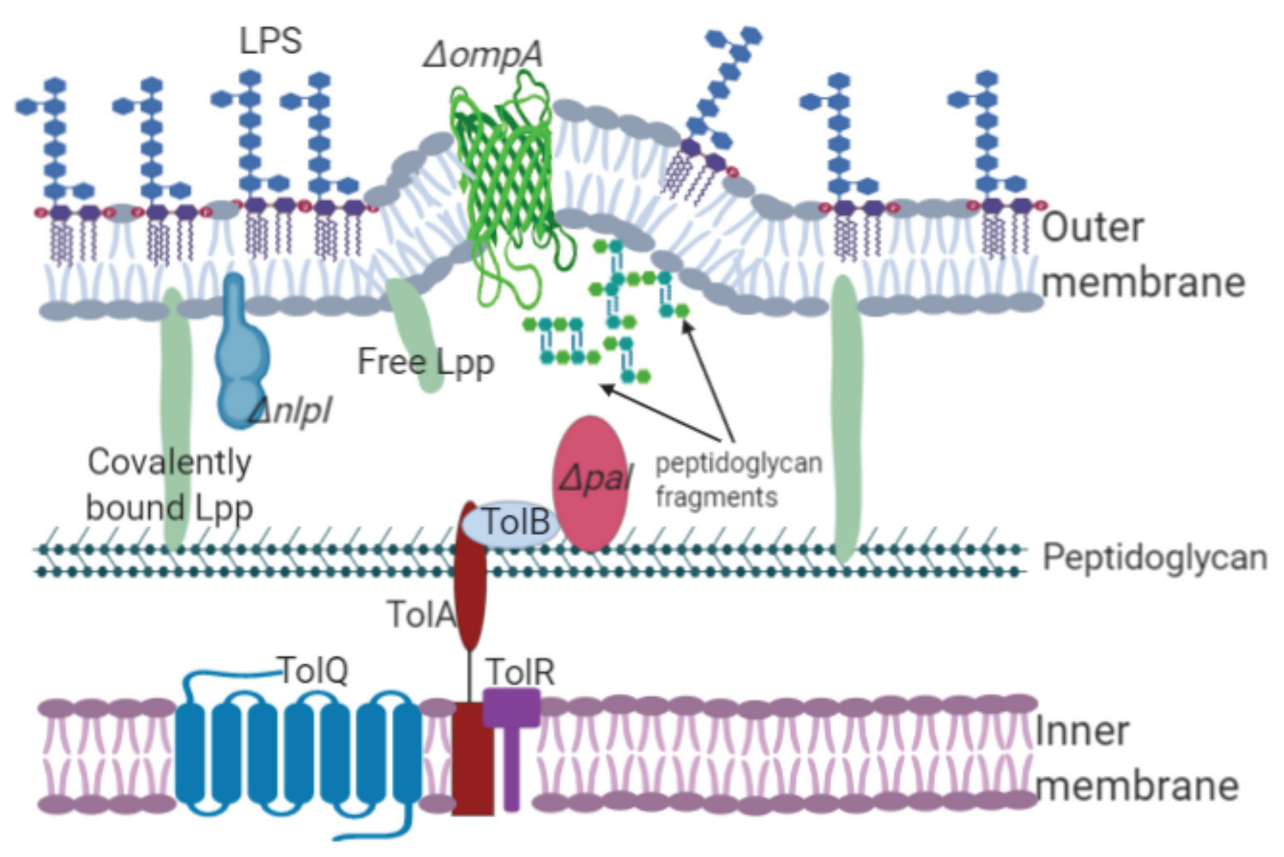

FIGURE 1 | Lipoproteins and outer membrane proteins are involved in OMV biogenesis. The Lpp, NIpl, OmpA, and Tol-Pal members maintain the stability of cellular envelopes joining the peptidoglycan layer with the inner membrane. Interruption or deletion of the genes encoding these proteins decrease the number of linkages, inducing OMV formation. For example, mutation of the pal gene decreases linkage with the outer membrane, leading to membrane protrusion and OMV release. Additionally, the accumulation of components in the periplasm, such as peptidoglycan precursors, triggers vesicle formation. 
of OmpA as a monomer or homodimer and its distribution on the outer membrane determine the sites where vesicles can form.

Deatherage et al. (2009) and Nevermann et al. (2019) treated S. typhi and S. typhimurium LT2 ompA mutants with deoxycholate together with vancomycin and evaluated bacterial viability. Nevermann et al. (2019) reported that the S. typhi ompA mutant was sensitive to deoxycholate and that the S. typhimurium ATCC14028s ompA mutant was sensitive to vancomycin. However, Deatherage et al. (2009) found that the S. typhimurium LT2 ompA mutant was not sensitive to deoxycholate. The sensitivity of the S. typhi and S. typhimurium ompA mutants to deoxycholate suggests defective outer membrane stability. Additionally, the hypervesiculating phenotype might be expressed because of defective outer membrane permeability. Although S. typhi and S. typhimurium are phylogenetically closely related, differences observed in the membrane stability of the mutants in the study suggest a different role for the OmpA lipoprotein in OMV biogenesis.

The accumulation of periplasmic compounds, including proteins and peptidoglycan, induces membrane protrusion and vesicle release (Hayashi et al., 2002; McBroom and Kuehn, 2007). Schwechheimer et al. (2014) analyzed the relationship among the accumulation of periplasmic compounds, Lpp linkages, and OMV release. The effects of the periplasmic content on OMV formation were analyzed using three E. coli mutants: (i) E. coli $\triangle a m p G \Delta a m i D$, a mutant defective in the transport of peptidoglycan-recycling residues; (ii) E. coli $\Delta r f a C, \Delta r f a G$, and $\triangle r f a P$ mutants defective in the transport and assembly of LPS; (iii) E. coli $\Delta \operatorname{deg} P$, a mutant that cannot degrade misfolded peptides in the periplasm. The hypervesiculation phenotype was exhibited by the three E. coli mutants likely because of the accumulation of peptidoglycan fragments, LPS, and proteins in the periplasm. However, the overall number of Lpp bonds were not affected because the levels of Lpp cross-linking were similar to those observed in the wild-type strain (Schwechheimer et al., 2014). By contrast, the E. coli $\Delta m e p A \Delta d a c B \Delta p b p G$ mutant, defective in three peptidoglycan hydrolases, showed a decreased number of Lpp-peptidoglycan linkages and increased OMV production (Schwechheimer et al., 2014). The vesiculation process was analyzed for $E$. coli strains displaying deficient genes encoding L,D-transpeptidases $(y n h G$ and $y c b B)$. ynhG (now renamed $l d t E$ ) and $y c b B(l d t D)$ encode enzymes that catalyze the formation of meso-diaminopimelyl $\rightarrow$ mesodiaminopimelyl crosslinks (also called DAP $\rightarrow$ DAP or 3-3 cross-links) in peptidoglycan (Magnet et al., 2007). The E. coli $\Delta y n h G \Delta y c b B$ mutant produced fewer OMVs than the wild-type strain, and the number of Lpp cross-links in this mutant increased (Schwechheimer et al., 2014). Therefore, the authors propose that the E. coli $\Delta y n h G \Delta y c b B$ mutant, lacking DAP-DAP linkages, exhibits Lpp and peptidoglycan cross-links formed randomly around the cell body, decreasing OMV release. These data revealed that the modulation of the peptidoglycan structure leads to a decrease in Lpp linkages and an increase in OMV production, while a decrease in OMV production is related to an increase in covalently bound Lpp to peptidoglycan (Schwechheimer et al., 2014).
Another lipoprotein found to be involved in OMV biogenesis is NlpA, which is an inner membrane lipoprotein. Mutation of this protein decreases OMV production in E. coli (Yamaguchi and Inouye, 1988; McBroom et al., 2006; Schwechheimer and Kuehn, 2013). Furthermore, Schwechheimer et al. (2014) observed that the single mutation of the inner-membrane-anchored lipoprotein NlpA decreased vesiculation in E. coli, while a specific mutation of the ompA gene increased OMV production. The E. coli $\triangle n l p A \triangle o m p A$ double mutant exhibited heightened vesiculation (Schwechheimer et al., 2014). However, the E. coli $\Delta y c f S \Delta y b i S \Delta e r f K \Delta n l p A$ mutant (defective in L,D-transpeptidases involved in the covalent crosslink between Lpp and peptidoglycan, and NlpA) showed increased vesiculation compared with that of the E. coli $\Delta y c f S \Delta y b i S \Delta e r f K$ mutant. The heightened vesiculation observed in both the E. coli $\triangle n l p A \triangle o m p A$ and E. coli $\Delta y c f \Delta y b i \Delta e r f K \Delta n l p A$ mutants can be explained in terms of NlpA, which provides stabilization of sites of the bacterial envelope necessary for Lpp and OmpA linkages (Schwechheimer et al., 2014). In 1998, Bernadac et al. used a Tol-Pal system mutated E. coli strain to purify and analyze OMVs using electron microscopy. The research team observed that the tolA, tolQ, and tolR mutants displayed a hypervesiculation phenotype, whereas the tolB and pal mutants showed a reduced vesicle production (Bernadac et al., 1998). The Tol-Pal system comprises proteins associated with outer membrane integrity. TolA, TolQ, and TolR link the inner membrane to peptidoglycan, whereas TolB and the peptidoglycan-associated lipoprotein (Pal) interact with the outer membrane (Gerding et al., 2007). McBroom et al. (2006) phenotyped random transposon mutants and found that the E. coli tolA, tolB, and pal mutants showed a hypervesiculation phenotype. Similar results of hypervesiculation were observed in the P. aeruginosa oprL and oprI mutants (Wessel et al., 2013). OprL and OprI are homologs to the Pal and Lpp proteins, respectively (Hancock et al., 1990). P. aeruginosa $o p r I$ and $o p r F$ mutants produced more OMVs than wild-type P. aeruginosa (Wessel et al., 2013). A recent study by Nevermann et al. (2019) identified genes related to OMV biogenesis in S. typhi employing transposon mutagenesis. Mutation of the tolR gene led to the expression of the hypervesiculation phenotype. The S. typhi tolR mutant was treated with deoxycholate together with vancomycin. Subsequently, cell viability was measured to evaluate whether membrane integrity had been affected. Following treatment with deoxycholate, neither cell growth nor hypervesiculation in the S. typhi tolR mutants was affected. These results indicated that OMV production was due to defective lipoprotein linkages. Tol-Pal members are highly homologous (possibly because of speciation events, gene duplication, and/or lipoprotein redundancy), and their distinct functions may be essential to OMV biogenesis.

NlpI is an outer-membrane-anchored lipoprotein whose function remains unknown. Although it was previously associated with cell division in E. coli, it has also been related to biofilm formation in S. typhimurium (Ohara et al., 1999). Inactivation of the nlpI gene in E. coli resulted in abnormal cell division and formation of membrane projections, whereas overexpression of this gene inhibited cell growth (Rouf et al., 2011; Banzhaf et al., 2020). The S. typhimurium ATCC14028s nlpI 
mutant expressed the hypervesiculation phenotype and was more resistant to vancomycin than the $S$. typhi nlpI mutant (Nevermann et al., 2019). However, the E. coli nlpI mutant displayed the hypervesiculation phenotype (McBroom et al., 2006). Schwechheimer et al. (2015) obtained an E. coli nlpI mutant displaying the hypervesiculation phenotype but no sensitivity to deoxycholate, concluding that the hypervesiculation phenotype observed was neither due to defects in membrane integrity nor cell lysis. NlpI regulates the activity of the peptidoglycan hydrolases Spr and PBP4, which play an essential role in cell wall renewal. Mutation of the $n l p$ I gene in E. coli increased Spr and PBP-4 activity, as well as peptidoglycan cleavage and peptidoglycan synthesis. The increased activity of Spr and PBP-4 hydrolyses observed in the E. coli nlpI mutant led to decreased linkages between Lpp and peptidoglycan and vesicle overproduction. Most likely, peptidoglycan dynamics (i.e., growth and renewal) are regulated by NlpI in the E. coli wild-type strain. However, peptidoglycan hydrolases Spr and PBP-4 decrease Lpp-peptidoglycan linkages, inducing outer membrane protrusion and subsequent OMV formation. Importantly, peptidoglycan dynamics and/or Lpp-peptidoglycan linkages are also affected by different hydrolases that are not regulated by NlpI. Based on these findings, NlpI lipoprotein is likely closely associated with OMV formation (Schwechheimer et al., 2015).

\section{Bacterial LPS Plays an Essential Role in OMV Production}

The outer membrane is an asymmetric structure comprising an inner leaflet made up of phospholipids along with proteins and an outer leaflet that contains phospholipids, proteins, and LPS. LPS is an essential structure for Gram-negative bacteria and is the most abundant antigen found on their cell surface; for example, the outer membrane of E. coli and Salmonella genera can contain up to 75\% LPS (Klein and Raina, 2019).

The structure of smooth LPS comprises an O-side chain, an intermediate region known as the core oligosaccharide and a glycolipid (lipid A) anchored to the outer membrane. Although smooth strains contain the O-side chain in their LPS, rough strains do not (Raetz and Whitfield, 2002). The core oligosaccharide is divided into two sections: the inner core, proximal to lipid $\mathrm{A}$, and the outer core, which is the attachment site for the $\mathrm{O}$-antigen. Lipid A can become chemically modified. For example, phosphoethanolamine, 4-amino-4-deoxy-Larabinose (L-Ara4N), and additional palmitate groups were added (Raetz and Whitfield, 2002). Such lipid A modifications make the bacteria more resistant to cationic antibacterial peptides and polymyxin (Raetz and Whitfield, 2002).

P. aeruginosa produces two variants of the $\mathrm{O}$-side chain antigen: the common polysaccharide antigen, also called the CPA or A-band (a short, neutrally charged molecule), together with the O-specific antigen, also referred to as the OSA or B-band (a highly immunogenic, negatively charged molecule; Lam et al., 2011). Both OSA and CPA were detected in OMVs obtained from a $P$. aeruginosa strain cultured in the presence and absence of gentamicin. OMVs exhibited a higher concentration of OSA in the absence of gentamicin; however, in the presence of this antibiotic, CPA was hardly detected within the vesicles (Kadurugamuwa and Beveridge, 1995). The addition of gentamicin (a polycation) to $P$. aeruginosa cultures modified electric charges in the outer membrane, affecting LPS packing into the OMVs. The role of negatively charged LPS in the biogenesis of OMVs was confirmed using P. aeruginosa $\mathrm{CPA}^{-}, \mathrm{OSAB}^{-}$, and $\mathrm{CPA}^{-} \mathrm{OSA}^{-}$mutants. In 2003, Nguyen et al. confirmed that the OSA ${ }^{-}$LPS complex also contributes to OMV formation because the P. aeruginosa $\mathrm{CPA}^{-}$mutant, which only synthesizes negatively charged LPS, released larger OMVs than those released by the $\mathrm{OSA}^{-}$mutant (Nguyen et al., 2003). These data showing that OMVs contain a higher concentration of negatively charged LPS suggest that OSA plays a role in vesicle formation.

In 2015, Cahill et al. while working with the Klebsiella pneumoniae wbbO mutant, a glycosyltransferase-defective strain lacking the O-side chain, reported that the release of OMVs was not affected, whereas, the outer membrane and vesicles from the $K$. pneumoniae $w b b O$ mutant exhibited a different protein profile and were quite distinct from the vesicles of the wild-type strain. Furthermore, vesicles released by the $K$. pneumoniae $w b b O$ mutant contained a higher concentration of proteins associated with posttranslational modification, protein turnover, and chaperones. However, OMVs produced by the $K$. pneumoniae wild-type strain contained proteins involved in cell wall, membrane, and envelope biosynthesis (Cahill et al., 2015). P. gingivalis, an etiologic agent of chronic periodontitis, also expresses two types of LPS, neutrally charged LPS (O-LPS) and negatively charged LPS (A-LPS; Paramonov et al., 2005, 2009). Studies performed on $P$. gingivalis porS and waaL $P$ mutants demonstrated that neither A-LPS nor O-LPS is essential for OMV biogenesis. The P. gingivalis porS mutant, lacking the flippase PorS, did not display the O-antigen in lipid A. However, the $P$. gingivalis waaL mutant lacks the $\mathrm{O}$-antigen ligase WaaL, generating rough cells. Nonetheless, the electrophoretic profile of proteins associated with OMVs produced by the $P$. gingivalis waal mutant was different from that obtained from the wildtype strain (Haurat et al., 2011). These results suggest that negatively charged LPS influence protein packing into OMVs.

In 2014, Murphy et al. analyzed lipid concentrations in OMVs released from P. aeruginosa $\mathrm{CPA}^{-}, \mathrm{OSA}^{-}$, and $\mathrm{CPA}^{-} \mathrm{OSA}^{-}$ mutants. No differences were found in either the concentration of lipids or number of vesicles released by all three mutants (Murphy et al., 2014). Most likely, repulsion between the core and lipid A produced an inducing effect on the $P$. aeruginosa $\mathrm{CPA}^{-} \mathrm{OSA}^{-}$double mutant but not on the CPA or OSA single mutant. By contrast, the double mutant exhibited an increased number of OMVs, but no change in size was observed. OMVs from the $P$. aeruginosa $\mathrm{CPA}^{-} \mathrm{OSA}^{-}$mutant and wild-type strain contain proteins sharing similar functions. Nevertheless, OMVs from the $P$. aeruginosa $\mathrm{CPA}^{-}$mutant showed the highest accumulation of proteins involved in the transport of small molecules. The P. aeruginosa $\mathrm{OSA}^{-}$mutant displayed the highest proportion of proteins involved in adaptation, protection, and transcriptional regulation (Murphy et al., 2014). A high content of periplasmic proteins and a low number of OMPs were detected in OMVs from the $P$. aeruginosa $\mathrm{OSA}^{-}$mutant. Conversely, high numbers of OMPs and periplasmic proteins 
were observed in OMVs from the $P$. aeruginosa $\mathrm{CPA}^{-} \mathrm{OSA}^{-}$ double mutant, $\mathrm{CPA}^{-}$mutant and wild-type strain (Murphy et al., 2014). These results suggest that OSA-LPS plays an important role in the selection of proteins. Proteins interacting with specific LPS lead to OMVs with different contents due to LPS composition. It is highly probable that the lack of the $\mathrm{O}$-side chain modifies the electric charge on the surface of the membrane, impairing electrostatic interactions between proteins and electrically charged LPS, and ultimately affecting the protein packing of OMVs.

PagL is an enzyme that modifies lipid A by removing the acyl chain at the 3-position of the disaccharide backbone (King et al., 2009). In S. typhimurium, a deacylated lipid A (i.e., penta-acylated lipid A) modified by PagL makes LPS less detectable to Toll-like receptor 4 of the mouse B-cell line (Kawasaki et al., 2004). PagL activity is regulated by a two-component system (represented as $\mathrm{PhoP} / \mathrm{PhoQ}$ ), $\mathrm{Mg}^{+2}$ and temperature, being less active at low $\mathrm{Mg}^{+2}$ concentrations and temperatures (Trent et al., 2001a; Ernst et al., 2006). OMVs from $P$. gingivalis displayed higher concentrations of deacylated lipids (Haurat et al., 2011). Therefore, the effect of lipid A deacylation on OMV biogenesis was evaluated using the expression of the PagL enzyme. The pagL gene was cloned using a low-copy expression vector lacking a control from the PhoP/PhoQ two-component system. S. typhimurium expressing PagL produced almost four times more OMVs than the wild-type strain (Elhenawy et al., 2016). The outer membrane of the S. typhimurium strain expressing PagL showed that hexa-acylated lipid A was predominant, while OMVs mainly contained penta-acylated lipid A (Elhenawy et al., 2016). The authors explained that these differences are likely based on conformational changes in the lipid A structure because hexa-acylated lipid A has a conical shape, while deacylated lipid A has a cylindrical or inverted cone shape. Thus, either the cylindrical or inverted-cone shape decreases hydrophobic interactions, leading to membrane protrusion and favoring OMV formation (Figure 2A; Schromm et al., 2000; Elhenawy et al., 2016). The presence of deacylated lipid A in other Gram-negative bacteria should be investigated to determine whether this is a common molecule implicated in OMV biogenesis. The quantitative comparison analysis of subtypes of LPS in OMVs and outer membranes of wild type S. typhimurium undergoing environmental shift conditions that do not rely on PagL supported an additional role for polymorphic regulation of membrane LPS composition in vesiculation (Bonnington and Kuehn, 2016). Nonlamellar types of lipids, which have an overall conical shape and a preference for the hexagonal phase, are thought to aid in the formation of nonbilayer structures. Accordingly, both the geometry and the propensity of individual membrane components to form nonbilayer lipid phases in the membrane are both likely to be important to vesiculation processes.

Citrobacter rodentium, a murine enteric pathogen, lacks the pagL gene. This bacterium uses two-component systems to modify LPS, and these systems may indirectly regulate OMV production, similar to PagL. One of these systems is $\mathrm{PmrAB}$, a two-component system regulated by low $\mathrm{pH}$ and
$\mathrm{Fe}^{+3}$ concentration. PmrAB regulates the expression of the pmrD gene. PmrB senses high $\mathrm{Fe}^{+3}$ concentrations. Once PmrA is activated through phosphorylation, it triggers the differential expression of $p m r C$ (also known as eptA) and $c p t A$; both $p m r C$ and $c p t A$ catalyze LPS modifications (Perez and Groisman, 2007). In Salmonella enterica, PmrC and CptA link phosphoethanolamine, while ArnT links 4-amino-4-deoxy-L arabinose to lipid A and the LPS core; these bonds are regulated by PmrAB (Trent et al., 2001b; Lee et al., 2004; Tamayo et al., 2005). Once 4-amino-4-deoxy-L arabinose and phosphoethanolamine are linked to lipid $\mathrm{A}$ and the core, the synthesis of negatively charged LPS is decreased, and molecules attached to LPS enhance resistance to antimicrobial peptides and oxidative stress (Sinha et al., 2019). The C. rodentium $\triangle c p t A, \triangle p m r C$, and $\triangle p m r A B$ mutants produce more OMVs than the wild-type strain (Sinha et al., 2019). Furthermore, decreased vesiculation was observed in C. rodentium strains overexpressing the $p m r C$ (eptA) or cptA genes, as shown in Figure 2B (Sinha et al., 2019). PmrC and CptA are putative transferases in C. rodentium; hence, mutations of these genes could lead to decreased modification of LPS with phosphoethanolamine, increasing the overall negative charge of LPS and causing outer-membrane curvature imbalance, subsequent membrane protrusion, and OMV release.

Nevermann et al. (2019) demonstrated that the waaC and $r f a E$ genes are also associated with OMV biogenesis in S. typhi. Additionally, RfaE has been associated with heptose (Hep) precursors necessary for inner-core LPS assembly in E. coli and S. typhimurium (Valvano et al., 2002). Heptoses are found in the oligosaccharide core, and they play a fundamental role in outer membrane stability and the crosslinking of adjacent LPS; they also interact with the positive charges of certain proteins (Heinrichs et al., 1998). Heptosyltransferase I (HepI or WaaC) catalyzes the addition of the first heptose to the inner Kdo (3-deoxy-D-manno-oct-2-ulosonic acid; Czyzyk et al., 2011). The S. typhi waaC mutant produced fewer vesicles than the wild-type strain, while the OMV protein profiles from both the mutant and wild-type strains were similar. Additionally, the S. typhi rfaE mutant produced more vesicles; however, when comparing OMV protein profiles from both the mutant and wild-type strains, relevant differences could clearly be observed. Lateral interactions with LPS molecules provide a significant fraction of the driving force necessary to establish lipid asymmetry in the outer membrane (Nikaido, 2003). Regarding the S. typhi rfaE mutant, it is likely that a defective link of heptose in the inner core alters its assembly, modifying certain interactions between positively and negatively charged LPS groups, and affecting the overall protein interaction with LPS. This finding could explain why the protein composition of OMVs from the S. typhi rfaE mutant was different from that obtained for OMVs from the wild-type strain. WaaC and $\mathrm{RfaE}$ induced an opposite effect on vesiculation. Mutation of the waaC gene led to the expression of the hypovesiculation phenotype, while mutation of the $r f a E$ gene induced a hypervesiculation phenotype. These data confirmed the same effects on the OMV phenotypes by waaC and $r f a E$ mutants found for E. coli (Kulp et al., 2015). Further research should 
A

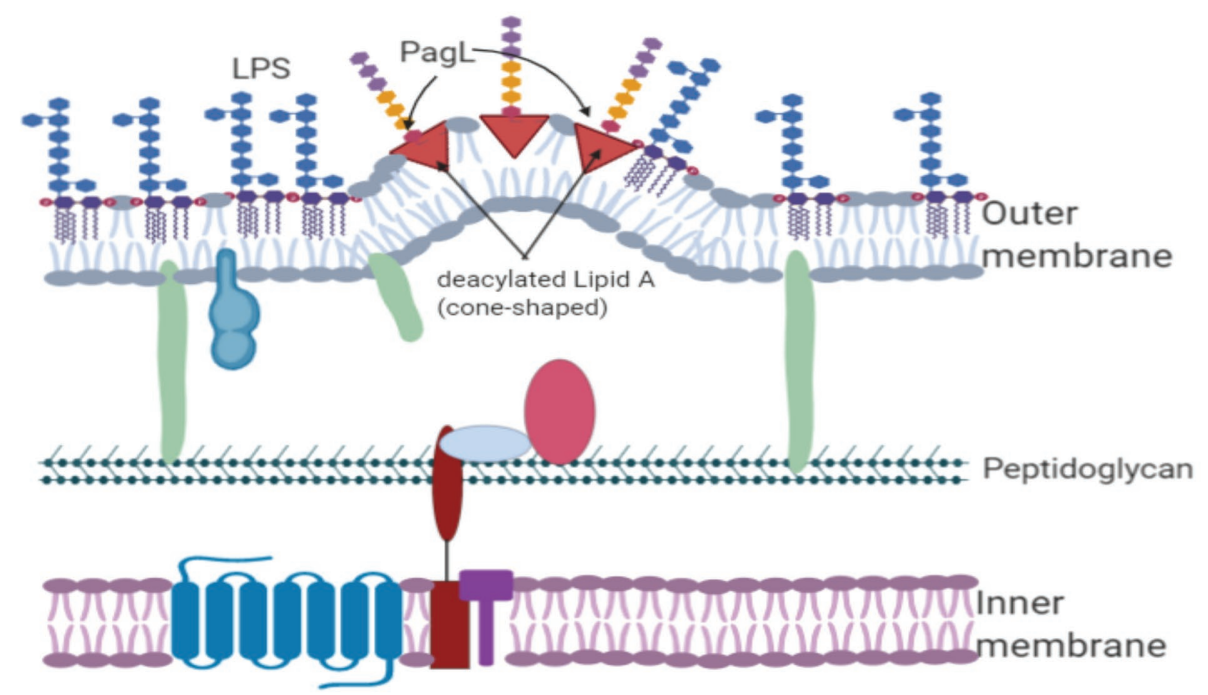

B
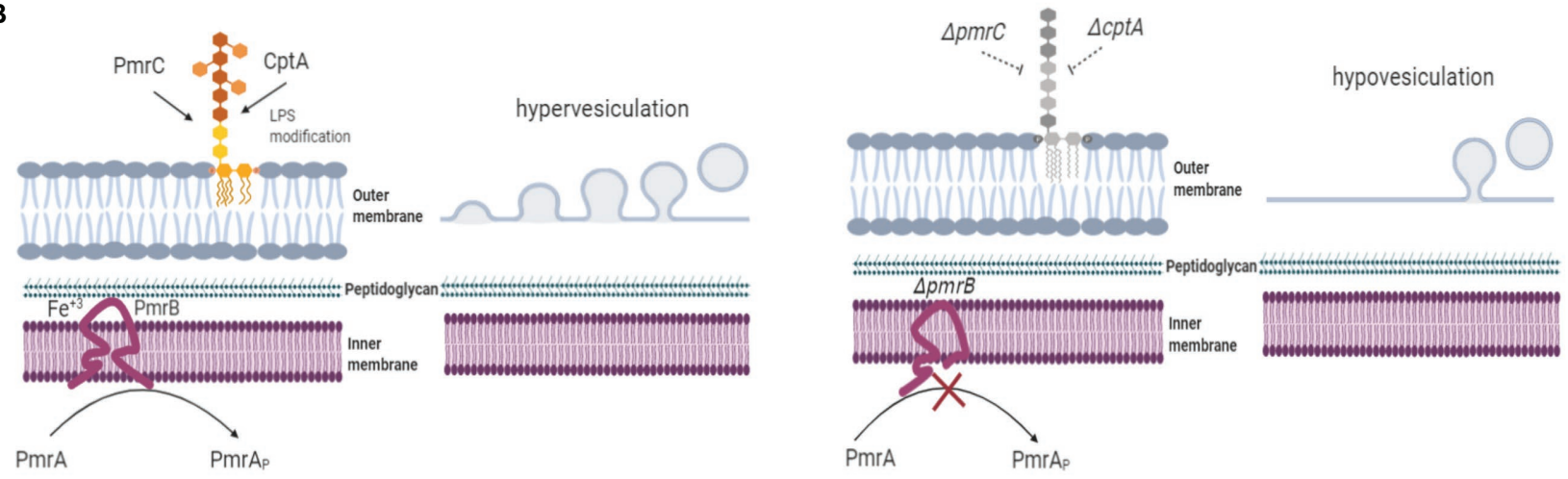

FIGURE 2 | LPS modifications are implicated in OMV release. Negatively charged LPS causes an outer membrane imbalance, subsequent membrane protrusion, and OMV release. LPS modifications also contribute to OMV formation. (A) The S. typhimurium strain expressing PagL without the PhoPQ control produces more OMVs than the wild-type strain. The outer membrane of $S$. typhimurium expressing PagL shows that hexa-acylated lipid $A$ is predominant, while OMVs primarily contain penta-acylated lipid A. Thus, either the cylindrical or inverted-cone shape decreases hydrophobic interactions, leading to membrane protrusion and favoring OMV formation. (B) Citrobacter rodentium uses a two-component system to modify LPS. PmrB, a member of this system, senses high Fe ${ }^{+3}$ concentrations and regulates the modification of lipid A. C. rodentium $\triangle p m r B$ cannot add groups to lipid A, leading to increased vesiculation.

focus on these enzymes, which are associated with inner core assembly, and how they contribute to OMV formation.

\section{Contribution of Phospholipids to OMV Biogenesis}

Most reports concerning OMV biogenesis focus on the role of lipoproteins and LPS. However, little is known about phospholipids, which are the major components of OMVs. The main phospholipids comprising the outer membrane of E. coli, in order of proportion, are phosphatidylethanolamine (PE), phosphatidylglycerol (PG), and cardiolipin (CL; Raetz and Dowhan, 1990). Using thin-layer chromatography, Horstman and Kuehn (2000) determined that the phospholipid composition of the outer membranes of the E. coli HB101 and ETEC 2 strains, as well as their respective vesicles, share similar components, including PE, PG, and CL. OMVs from Actinobacillus actinomycetemcomitans displayed higher $\mathrm{CL}$ and $\mathrm{PE}$ concentrations and a low proportion of unidentified lipids, which are not present in the outer membrane (Kato et al., 2002). OMVs from $P$. aeruginosa showed high $P G$ and stearic acid concentrations, but a low percentage of unsaturated fatty acids released from vesicles composed of a rigid membrane (Tashiro et al., 2011). The orf5 and plsC1 genes of Shewanella livingstonesis play an essential role in eicosapentaenoic acid biosynthesis, while plsC1 and plsC4 code for acyltransferases that are involved in the synthesis of membrane phospholipids. Mutation of orf5, plsC1, and plsC4 in S. livingstonesis increased the production of vesicles (Yokoyama et al., 2017).

Gram-negative bacteria maintain asymmetric distribution of phospholipids in their membranes through the translocation 
of phospholipids from the inner membrane to the inner leaflet of the outer membrane (known as phospholipid anterograde transport) and inversely (known as phospholipid retrograde transport; Shrivastava and Chng, 2019). Regarding anterograde transport, the molecular determinants implicated in the process are still not fully understood; however, the PbgA/YejM lipoprotein is involved in CL transport from the inner to the outer membrane in Shigella flexneri and S. typhimurium (Dalebroux et al., 2015; Rossi et al., 2017). Regarding retrograde transport, two systems have been described: (i) the Tol-Pal system is involved in the movement of phospholipids from the inner leaflet of the outer membrane to the inner membrane; (ii) the OmpC-Mla system is responsible for outer membrane asymmetry by transporting misplaced phospholipids found in the outer leaflet of the outer membrane to the inner membrane (Chong et al., 2015; Shrivastava et al., 2017). The MlaA lipoprotein interacts with $\mathrm{OmpC}$, which is embedded in the outer membrane and removes phospholipids in the outer leaflet of the outer membrane to another component of the system the $\mathrm{MlaC}$ protein. Subsequently, $\mathrm{MlaC}$ delivers these phospholipids to the MlaFEDB complex located in the inner membrane. This complex can then reintegrate these phospholipids to the inner membrane (Malinverni and Silhavy, 2009; Chong et al., 2015). Mutation of mla in E. coli induces vesicle production at the septa, increases hepta-acylated LPS, and promotes cell death (Sutterlin et al., 2016). Vesicle overproduction decreases the lipid levels in the outer membrane that are then replaced by lipids from the inner membrane. The decrease in lipids of the inner membrane leads to cell lysis (Sutterlin et al., 2016).

Roier et al. (2016) mutated the vacJ and yrbE genes of Haemophilus influenzae, which are homologous to the mlaA and mlaE genes (respectively) of $E$. coli and are involved in lipid membrane asymmetry. Vesiculation increased 1.6- and 2.2 -fold in the H. influenzae vacJ and $y r b E$ mutants, respectively, while vesicle production decreased in the complemented mutants. Vesicles from the H. influenzae vacj and yrbE mutants exhibited higher levels of PE and myristic acid (C14:0), similar to the outer membrane composition in the wild-type strain; however, the PE and C14:0 fatty acid ratio increased only in vesicles produced by the mutants. Hypervesiculation in $H$. influenzae vacJ and $y r b E$ was directly due to phospholipid accumulation in the outer membrane because the genes involved in retrograde transport were mutated. The $H$. influenzae vacJ and $y r b E$ mutants showed the hypervesiculation phenotype, which could be caused by the regulation of the transport of phospholipids from the inner leaflet to the outer leaflet of the outer membrane. Mutation of the homologous genes vacJ and $y r b E$, which are associated with phospholipid transport in V. cholerae, E. coli, and Campylobacter jejuni, increased vesiculation, confirming the hypothesis that phospholipid asymmetry (retrograde trafficking of phospholipids) plays a role in OMV biogenesis (Roier et al., 2016; Davies et al., 2019). Homologous proteins to the phospholipid $\mathrm{ABC}$ transport system were found in phylogenetically distant species, such as Pasteurella multocida, P. aeruginosa, S. typhimurium, and Yersinia enterocolitica, among others. This finding suggests that the mechanism of OMV formation may be strongly linked to phospholipid transport (Roier et al., 2016). To trigger vesicle formation in the outer membrane, the transport of phospholipids from the inner membrane to the outer membrane should be significantly faster than that from the outer membrane to the inner membrane. Disorganization in phospholipid transport leads to phospholipid accumulation in the outer membrane, provoking OMV release (Figure 3).

Differences found in the composition of these phospholipids suggest that vesicles are released from the microdomains of diverse phospholipid compositions. However, the possibility of a different mechanism that can regulate phospholipids in OMVs should not be excluded. The composition of predominant phospholipids in OMVs has already been determined. For example, vesicles purified from E. coli ETEC exhibited high concentrations of PG, CL, and PE. By contrast, OMVs from A. actinomycetemcomitans contained high concentrations of $\mathrm{CL}$ and $\mathrm{PE}$, whereas OMVs from $P$. aeruginosa only showed high concentrations of PG, and OMVs from $H$. influenzae mainly comprised PE (Kato et al., 2002). The accumulation of certain lipids, such as PG and PE, in OMVs is essential to decipher a mechanism for OMV biogenesis.

Tol-Pal helps maintain outer membrane stability through lipoprotein linkages. This system is involved in phospholipid retrograde transport and contributes to OMV biogenesis (Masilamani et al., 2018; Shrivastava and Chng, 2019). Because the S. typhimurium tolA, tolQ, and tolR mutants display high levels of PG and PE in the outer membrane, the presence of glycerophospholipids may promote OMV release (Masilamani et al., 2018). Because Tol-Pal proteins lack a phospholipidbinding domain, they may stabilize unidentified phospholipid transporters or act as phospholipid transporters from the inner to the outer membrane (Shrivastava and Chng, 2019).

\section{Bacterial Flagellin Induces OMV Release}

Several studies have shown an association between the bacterial flagellum and OMVs. The flagellum comprises a basal body, a flexible linker known as the hook, and a filament that drives the bacterium's movement. The filament of the bacterial flagellum comprises the flagellin protein (Chen et al., 2011). Using a density gradient protocol, OMVs from E. coli were purified, and flagellin (FliC) was identified using proteomics (Lee et al., 2007). Manabe et al. analyzed vesicles from E. coli W3110 and the E. coli $\Delta f l i C$ mutant derived from the W3110 strain. OMVs were obtained using a density gradient protocol. Both flagellin and OMVs from the wild-type strain were found in the same fraction collected from the density gradient. Moreover, flagellin was detected in the lumen of fractioned vesicles from the wildtype strain, and only in the mutant did OMV production decrease (Manabe et al., 2013). In another study, researchers tried to correlate the presence of flagella and OMV production in Vibrio fischeri; in this bacterium, the flagellum shaft is encased by a membranous sheath (Millikan and Ruby, 2004; Aschtgen et al., 2016). In this study, vesicle production was determined in the $V$. fischeri motB1 mutant (MotB1 is a sodium pump and the main motor protein for the flagellum machinery), $V$. fischeri nonflagellated flrA mutant (flrA codes for a transcriptional activator), $V$. fischeri hyperflagellated swarmer strain (expresses 3- to 4-fold more flagella), and V. fischeri wild-type strain. 


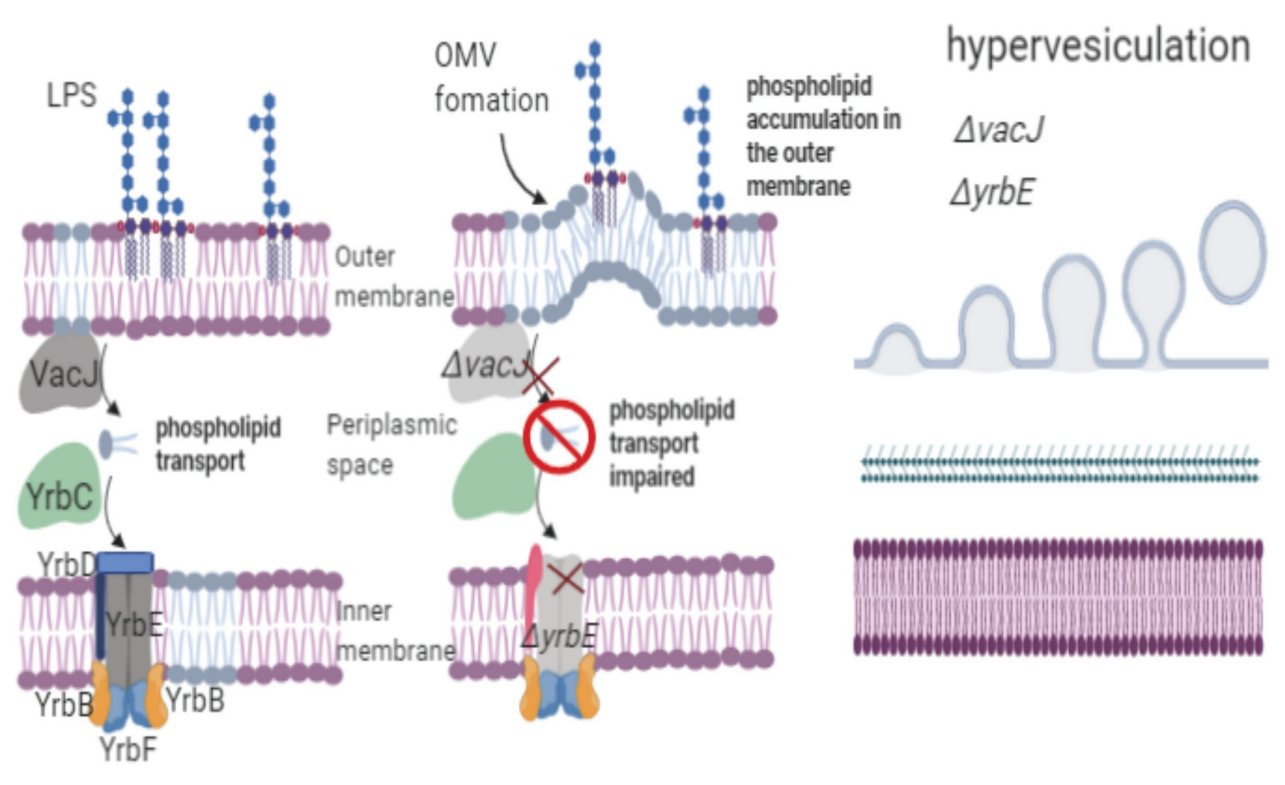

FIGURE 3 | Phospholipid transport regulates OMVs biogenesis. H. influenzae vacJ and yrbE mutants show a hypervesiculation phenotype because of the accumulation of phospholipids in the outer membrane. The vacJ and yrbE genes are involved in retrograde transport, maintaining membrane asymmetry. To trigger vesicle formation in the outer membrane, the transport of phospholipids from the inner membrane to the outer membrane should be significantly faster than phospholipid transport from the outer membrane to the inner membrane. Disorganization in phospholipid transport leads to the accumulation of phospholipids in the outer membrane, provoking OMV release.

However, the number of vesicles decreased in the V. fischeri flrA mutant that lacks flagella. Vesicle production increased in the hyperflagellated strain. The addition of phenamil (a sodiumpump blocking reagent) to the $V$. fischeri hyperflagellated strain decreased OMV production, suggesting that flagellum rotation improves vesicle release (Aschtgen et al., 2016). To demonstrate the association of flagella with OMV production, the following organisms were analyzed: $V$. fischeri and $V$. parahaemolyticus (which expresses several sheathed flagella), V. cholerae (which contains unique polar sheathed flagella), E. coli (which expresses an unsheathed flagellum), and the $V$. fischeri nonflagellated flrA mutant. $V$. fischeri and $V$. parahaemolyticus released more vesicles than V. cholerae and E. coli, while the V. cholerae nonflagellated mutant exhibited decreased OMV production (Aschtgen et al., 2016). They concluded that species expressing flagella such as E. coli and Vibrio released more vesicles. Clearly, unsheathed and sheathed flagella perturb the outer membrane and promote OMV release. To better ascertain the relationship between flagella and OMV production, more flagellated strains along with their corresponding nonflagellated mutants and hyperflagellated strains should be studied. It is important to explore whether flagellum movement only promotes the release of vesicles from the surrounding outer membrane area or promotes vesiculation from the whole cell body.

\section{Pseudomonas Quinolone Signaling and Its Role in OMV Biogenesis}

Bacterial species such as $P$. aeruginosa regulate the secretion of virulence factors by sensing bacterial density through the quorum-sensing mechanism (Strateva and Mitov, 2011).
P. aeruginosa produces a quinolone called the Pseudomonas quinolone signal (PQS). To regulate the expression of some genes, PQS binds to the PqsR receptor (Lin et al., 2018). The synthesis of PQS is regulated by pqsABCDE along with other genes, including $p h n A B$ and $p q s H$. PqsA triggers PQS synthesis. $\mathrm{PqsB}, \mathrm{PqsC}$, and PqsD then produce 2-heptyl-4-quinolone (HHQ) or quinolone and other precursors. Finally, $\mathrm{PqsH}$ catalyzes the conversion of HHQ into PQS. The P. aeruginosa pqsH mutant synthesizes quinolones but not PQS (Lin et al., 2018).

The quorum-sensing system in $P$. aeruginosa comprises quinolone molecules, including 3-oxo-dodecanoyl homoserine lactone (3OC12-HSL), butyryl homoserine lactone (C4-HSL), and 2-heptyl-3-hydroxy-4-quinolone (PQS; Déziel et al., 2004). OMVs from $P$. aeruginosa contain higher PQS (86\%) than 12-HSL and C4-HSL, which were measured using thin-layer chromatography (TLC) and mass spectrometry (Mashburn-Warren and Whiteley, 2005). Additionally, the $P$. aeruginosa pqsA mutant (displaying defective quinolone synthesis) showed a 10-fold decrease in OMV production. Vesicle production was restored after treating mutants with a synthetic quinolone. Thus, PQS may also be involved in OMV release. However, when the $P$. aeruginosa $m v f R$ mutant (which lacks the PQS $m v f R$ regulator) was treated with exogenous PQS, it yielded the same number of vesicles as that of the wild-type strain. This finding suggests physical interactions among $\mathrm{PQS}$ and different undetermined molecules found in outer membrane sites where it begins to bulge (Mashburn-Warren and Whiteley, 2005). Additionally, the interaction of LPS with either PQS or the alkyl chain and hydroxyl group $(-\mathrm{OH})$ of $\mathrm{PQS}$ induced a curvature in the outer membrane necessary to start vesicle formation. Hence, a synthetic 
compound similar to PQS (which lacks the alkyl chain and hydroxyl group) does not interact with LPS, while the natural PQS molecule interacts with LPS (Mashburn-Warren et al., 2008). Moreover, PQS could impair the interaction among divalent cations $\left(\mathrm{Mg}^{+2}\right.$ and $\left.\mathrm{Ca}^{+2}\right)$ and phosphate at the 4-position of lipid A, affecting the motility of lipid A and destabilizing the outer membrane, improving outer membrane protrusion and vesicle release (Bredenbruch et al., 2006; Mashburn-Warren et al., 2008).

In 2012, Schertzer and Whiteley proposed a novel bilayercoupled model to explain OMV biogenesis, which they theorized could be mediated by PQS in P. aeruginosa (Schertzer and Whiteley, 2012). This model requires the presence of an amphiphilic molecule that, due to specific electric interactions, would concentrate in the outer leaflet of the membrane, leading to the expansion of the outer leaflet while promoting membrane curvature (Sheetz and Singer, 1974). To study the effect of small molecules on the cell membrane, a solution prepared with a low concentration of PQS was added to red blood cells. The erythrocytes showed crenation and membrane protrusions, an effect similar to that elicited during vesicle production (Schertzer and Whiteley, 2012). The addition of chlorpromazine, which induces cup formation in red blood cells, prevented the formation of protrusions in the membrane. Such evidence indicates that PQS may contribute to OMV release, in accordance with the bilayercoupled model proposed by Schertzer and Whiteley (2012).

P. aeruginosa PA01 strain was previously shown to produce a higher concentration of $\mathrm{PQS}$ in the inner membrane and released fewer OMVs. By contrast, PQS production in P. aeruginosa PA14 was extracellular, and a higher OMV production was observed. These results suggest that a relevant difference between the strains could be defective PQS export (Florez et al., 2017). Temporal analyses showed PQS accumulation in the inner membrane of the $P$. aeruginosa PA01 strain due to PQS saturation. This saturation was observed after culturing the strain for $10 \mathrm{~h}$ in Luria-Bertani medium (Florez et al., 2017). When the PA01 strain was cultured using brain infusion broth, vesicle production increased along with PQS export compared with that obtained in Luria-Bertani medium. These results were similar to those reported in clinical strains of $P$. aeruginosa isolated from patients with cystic fibrosis. These strains showed an increase in OMV production and PQS export in brain infusion broth culture (Florez et al., 2017). Future research is required to analyze PQS transport in Gram-negative clinical strains and define possible PQS-associated mechanisms in OMV biogenesis.

In 2018, Horspool and Schertzer treated different strains of gammaproteobacteria, such as E. coli, Klebsiella pneumoniae, and Proteus mirabilis, with exogenous PQS. All the strains increased vesicle production up to 3.5 -fold. This effect was not observed in alphaproteobacteria such as Agrobacterium tumefaciens and Caulobacter crescentus. Importantly, the authors observed an increase in OMV production when using the $P$. aeruginosa strain that was stimulated with a concentrated supernatant from cultures of E. coli and
K. pneumoniae (Horspool and Schertzer, 2018). Molecules present in the supernatants induced OMV production in $P$. aeruginosa, but these molecules have yet to be identified. Although Mashburn-Warren and Whiteley attributed OMV production in $P$. aeruginosa exclusively to $\mathrm{PQS}$, they also observed a minimal concentration of acyl-homoserine lactone packaged in OMVs. Therefore, exogenous acyl-homoserine lactone from E. coli and K. pneumoniae induce membrane curvature, leading to OMV release in P. aeruginosa (MashburnWarren and Warren, 2005; Schertzer and Whiteley, 2012).

\section{CONCLUDING REMARKS}

Several works have focused on explaining the different possible OMV biogenesis mechanisms. Lipoproteins, outer membrane proteins, LPS, and flagellin have been proposed as the key pieces in different mechanisms of OMV biogenesis.

Lipoproteins and outer membrane proteins involved in linkages within the bacterial envelopes regulate membrane protrusion and subsequent OMV release. Further investigation is needed and should focus on the search for lipoproteins and outer membrane proteins homologous to those reviewed here involved in OMV biogenesis. The interaction with the peptidoglycan and/or outer membrane and null mutants were then analyzed to determine their role in vesicle formation. It is possible that more than one molecule, such as LPS, outer membrane proteins, and phospholipids, could contribute to vesicle formation. Although LPS differs considerably among bacterial species, it has also been associated with OMV biogenesis. LPS charge and architecture are involved in OMV formation and protein composition. In addition, it should be explored which LPS component (O-antigen, core, and/ or lipid A) plays the major role in vesiculation. However, retrograde phospholipid transport is involved in OMV release; phospholipid accumulation increases outer membrane protrusion and subsequent vesicle release. Other molecules, including PQS or flagellin, could be involved in vesicle formation. Although the secretion of PQS molecules or presence of flagella in the cell body is restricted to a few bacterial species, their role in vesiculation must be explored in more detail.

\section{AUTHOR CONTRIBUTIONS}

All authors listed have made a substantial, direct and intellectual contribution to the work, and approved it for publication.

\section{FUNDING}

This work was funded by CONACYT 61529, SIP-IPN 20182152, 20195737, 20200594, and SAGARPA-CONACYT 2017-02-291311. EA-C was supported by CONACYT and PIFI-IPN scholarships. MA-A, ER, ZG-L, and AC-R were supported by fellowships from COFAA-IPN, SIP-EDI, and SNI-CONACYT. 


\section{REFERENCES}

Aschtgen, M. S., Lynch, J. B., Koc, E., Schwartzman, J., McFall-Ngai, M., and Ruby, E. (2016). Rotation of Vibrio fischeri flagella produces outer membrane vesicles that induce host development. J. Bacteriol. 198, 2156-2165. doi: 10.1128/JB.00101-16

Avila-Calderón, E. D., Araiza-Villanueva, M. G., Cancino-Diaz, J. C., López-Villegas, E. O., Sriranganathan, N., Boyle, S. M., et al. (2015). Roles of bacterial membrane vesicles. Arch. Microbiol. 197, 1-10. doi: 10.1007/ s00203-014-1042-7

Banzhaf, M., Yau, H. C., Verheul, J., Lodge, A., Kritikos, G., Mateus, A., et al. (2020). Outer membrane lipoprotein NlpI scaffolds peptidoglycan hydrolases within multi-enzyme complexes in Escherichia coli. EMBO J. 39:e102246. doi: $10.15252 / \mathrm{embj} .2019102246$

Bernadac, A., Gavioli, M., Lazzaroni, J. C., Raina, S., and Lloubès, R. (1998). Escherichia coli tol-pal mutants form outer membrane vesicles. J. Bacteriol. 180, 4872-4878. doi: 10.1128/JB.180.18.4872-4878.1998

Bonnington, K. E., and Kuehn, M. J. (2014). Protein selection and export via outer membrane vesicles. Biochim. Biophys. Acta. 1843, 1612-1619. doi: 10.1016/j.bbamcr.2013.12.011

Bonnington, K. E., and Kuehn, M. J. (2016). Outer membrane vesicle production facilitates LPS remodeling and outer membrane maintenance in Salmonella during environmental transitions. MBio 7, e01532-e01616. doi: 10.1128/ mBio.01532-16

Bredenbruch, F., Geffers, R., Nimtz, M., Buer, J., and Häussler, S. (2006). The Pseudomonas aeruginosa quinolone signal ( $\mathrm{PQS})$ has an iron-chelating activity. Environ. Microbiol. 8, 1318-1329. doi: 10.1111/j.1462-2920.2006.01025.x

Cahill, B. K., Seeley, K. W., Gutel, D., and Ellis, T. N. (2015). Klebsiella pneumoniae $\mathrm{O}$ antigen loss alters the outer membrane protein composition and the selective packaging of proteins into secreted outer membrane vesicles. Microbiol. Res. 180, 1-10. doi: 10.1016/j.micres.2015.06.012

Chen, S., Beeby, M., Murphy, G. E., Leadbetter, J. R., Hendrixson, D. H., Briegel, A., et al. (2011). Structural diversity of bacterial flagellar motors. EMBO J. 30, 2972-2981. doi: 10.1038/emboj.2011.186

Chong, Z. S., Woo, W. F., and Chng, S. S. (2015). Osmoporin OmpC forms a complex with MlaA to maintain outer membrane lipid asymmetry in Escherichia coli. Mol. Microbiol. 98, 1133-1146. doi: 10.1111/mmi.13202

Czyzyk, D. J., Liu, C., and Taylor, E. A. (2011). Lipopolysaccharide biosynthesis without the lipids: recognition promiscuity of Escherichia coli heptosyltransferase I. Biochemistry 50, 10570-10572. doi: 10.1021/bi201581b

Dalebroux, Z. D., Edrozo, M. B., Pfuetzner, R. A., Ressl, S., Kulasekara, B. R., Blanc, M. -P., et al. (2015). Delivery of cardiolipins to the Salmonella outer membrane is necessary for survival within host tissues and virulence. Cell Host Microbe 17, 441-451. doi: 10.1016/j.chom.2015.03.003

Davies, C., Taylor, A. J., Elmi, A., Winter, J., Liaw, J., Grabowska, A. D., et al. (2019). Sodium taurocholate stimulates Campylobacter jejuni outer membrane vesicle production via down-regulation of the maintenance of lipid asymmetry pathway. Front. Cell. Infect. Microbiol. 9:177. doi: 10.3389/ fcimb.2019.00177

Deatherage, B. L., Lara, J. C., Bergsbaken, T., Rassoulian Barrett, S. L., Lara, S., and Cookson, B. T. (2009). Biogenesis of bacterial membrane vesicles. Mol. Microbiol. 72, 1395-1407. doi: 10.1111/j.1365-2958.2009.06731.x

Déziel, E., Lépine, F., Milot, S., He, J., Mindrinos, M. N., Tompkinset, R. G., et al. (2004). Analysis of Pseudomonas aeruginosa 4-hydroxy-2-alkylquinolines (HAQs) reveals a role for 4-hydroxy-2-heptylquinoline in cell-to-cell communication. Proc. Natl. Acad. Sci. U. S. A. 101, 1339-1344. doi: 10.1073/ pnas. 0307694100

Eddy, J. L., Gielda, L. M., Caulfield, A. J., Rangel, S. M., and Lathem, W. W. (2014). Production of outer membrane vesicles by the plague pathogen Yersinia pestis. PLoS One 9:e107002. doi: 10.1371/journal.pone.0107002

Elhenawy, W., Bording-Jorgensen, M., Valguarnera, E., Haurat, M. F., Wine, E., and Feldman, M. F. (2016). LPS remodeling triggers formation of outer membrane vesicles in Salmonella. MBio 7, e00940-e01016. doi: 10.1128/ mBio.00940-16

Ernst, R. K., Adams, K. N., Moskowitz, S. M., Kraig, G. M., Kawasaki, K., Stead, C. M., et al. (2006). The Pseudomonas aeruginosa lipid a deacylase: selection for expression and loss within the cystic fibrosis airway. J. Bacteriol. 188, 191-201. doi: 10.1128/JB.188.1.191-201.2006
Florez, C., Raab, J. E., Cooke, A. C., and Schertzer, J. W. (2017). Membrane distribution of the Pseudomonas quinolone signal modulates outer membrane vesicle production in Pseudomonas aeruginosa. MBio 8, e01034-e01117. doi: 10.1128/mBio.01034-17

Gamazo, C., and Moriyón, I. (1987). Release of outer membrane fragments by exponentially growing Brucella melitensis cells. Infect. Immun. 55, 609-615. doi: 10.1128/IAI.55.3.609-615.1987

Gerding, M. A., Ogata, Y., Pecora, N. D., Niki, H., and De Boer, P. A. (2007). The trans-envelope Tol-Pal complex is part of the cell division machinery and required for proper outer-membrane invagination during cell constriction in E. coli. Mol. Microbiol. 63, 1008-1025. doi: 10.1111/j.1365-2958.2006.05571.x

Grenier, D., and Bélanger, M. (1991). Protective effect of Porphyromonas gingivalis outer membrane vesicles against bactericidal activity of human serum. Infect. Immun. 9, 3004-3008. doi: 10.1128/IAI.59.9.3004-3008.1991

Hancock, R. E., Siehnel, R., and Martin, N. (1990). Outer membrane proteins of Pseudomonas. Mol. Microbiol. 4, 1069-1075. doi: 10.1111/j.1365-2958.1990. tb00680.x

Haurat, M. F., Aduse-Opoku, J., Rangarajan, M., Dorobantu, L., Gray, R. M., Curtis, M. A., et al. (2011). Selective sorting of cargo proteins into bacterial membrane vesicles. J. Biol. Chem. 286, 1269-1276. doi: 10.1074/jbc. M110.185744

Hayashi, J., Hamada, N., and Kuramitsu, H. K. (2002). The autolysin of Porphyromonas gingivalis is involved in outer membrane vesicle release. FEMS Microbiol. Lett. 216, 217-222. doi: 10.1111/j.1574-6968.2002.tb11438.x

Heinrichs, D. E., Yethon, J. A., and Whitfield, C. (1998). Molecular basis for structural diversity in the core regions of the lipopolysaccharides of Escherichia coli and Salmonella enterica. Mol. Microbiol. 30, 221-232. doi: 10.1046/j.1365-2958.1998.01063.x

Hoekstra, D., van der Laan, J. W., de Leij, L., and Witholt, B. (1976). Release of outer membrane fragments from normally growing Escherichia coli. Biochim. Biophys. Acta 455, 889-899. doi: 10.1016/0005-2736(76)90058-4

Horspool, A. M., and Schertzer, J. W. (2018). Reciprocal cross-species induction of outer membrane vesicle biogenesis via secreted factors. Sci. Rep. 8:9873. doi: 10.1038/s41598-018-28042-4

Horstman, A. L., and Kuehn, M. J. (2000). Enterotoxigenic Escherichia coli secretes active heat-labile enterotoxin via outer membrane vesicles. J. Biol. Chem. 275, 12489-12496. doi: 10.1074/jbc.275.17.12489

Jan, A. T. (2017). Outer membrane vesicles (OMVs) of gram-negative bacteria: a perspective update. Front. Microbiol. 8:1053. doi: 10.3389/fmicb.2017.01053

Jin, J. S., Kwon, S. O., Moon, D. C., Gurung, M., Lee, J. H., Kim, S. I., et al. (2011). Acinetobacter baumannii secretes cytotoxic outer membrane protein a via outer membrane vesicles. PLoS One 6:e17027. doi: 10.1371/journal. pone. 0017027

Kadurugamuwa, J. L., and Beveridge, T. J. (1995). Virulence factors are released from Pseudomonas aeruginosa in association with membrane vesicles during normal growth and exposure to gentamicin: a novel mechanism of enzyme secretion. J. Bacteriol. 177, 3998-4008. doi: 10.1128/jb.177.14.3998-4008.1995

Kato, S., Kowashi, Y., and Demuth, D. R. (2002). Outer membrane-like vesicles secreted by Actinobacillus actinomycetemcomitans are enriched in leukotoxin. Microb. Pathog. 32, 1-13. doi: 10.1006/mpat.2001.0474

Katsui, N., Tsuchido, T., Hiramatsu, R., Fujikawa, S., Takano, M., and Shibasaki, I. (1982). Heat-induced blebbing and vesiculation of the outer membrane of Escherichia coli. J. Bacteriol. 151, 1523-1531. doi: 10.1128/JB.151.3.15231531.1982

Kawasaki, K., Ernst, R. K., and Miller, S. I. (2004). 3-O-deacylation of lipid a by PagL, a PhoP/PhoQ-regulated deacylase of Salmonella typhimurium, modulates signaling through toll-like receptor 4. J. Biol. Chem. 279, 20044-20048. doi: $10.1074 /$ jbc.M401275200

King, J. D., Kocíncová, D., Westman, E. L., and Lam, J. S. (2009). Review: lipopolysaccharide biosynthesis in Pseudomonas aeruginosa. Innate Immun. 15, 261-312. doi: 10.1177/1753425909106436

Klein, G., and Raina, S. (2019). Regulated assembly of LPS, its structural alterations and cellular response to LPS defects. Int. J. Mol. Sci. 20:E356. doi: $10.3390 /$ ijms 20020356

Knox, K. W., Vesk, M., and Work, E. (1966). Relation between excreted lipopolysaccharide complexes and surface structures of a lysine-limited culture of Escherichia coli. J. Bacteriol. 92, 1206-1217. doi: 10.1128/JB.92.4. 1206-1217.1966 
Koike, M., Iida, K., and Matsuo, T. (1969). Electron microscopic studies on mode of action of polymyxin. J. Bacteriol. 97, 448-452. doi: 10.1128/ JB.97.1.448-452.1969

Kulp, A. J., Sun, B., Ai, T., Manning, A. J., Orench-Rivera, N., Schmid, A. K., et al. (2015). Genome-wide assessment of outer membrane vesicle production in Escherichia coli. PLoS One 10:e0139200. doi: 10.1371/journal.pone.0139200

Lam, J. S., Taylor, V. L., Islam, S. T., Hao, Y., and Kocíncová, D. (2011). Genetic and functional diversity of Pseudomonas aeruginosa lipopolysaccharide. Front. Microbiol. 2:118. doi: 10.3389/fmicb.2011.00118

Lee, E. Y., Bang, J. Y., Park, G. W., Choi, S. C., Seoun, J., Kim, H. -J., et al. (2007). Global proteomic profiling of native outer membrane vesicles derived from Escherichia coli. Proteomics 7, 3143-3153. doi: 10.1002/pmic.200700196

Lee, H., Hsu, F. F., Turk, J., and Groisman, E. A. (2004). The PmrA-regulated pmrC gene mediates phosphoethanolamine modification of lipid a and polymyxin resistance in Salmonella enterica. J. Bacteriol. 186, 4124-4133. doi: $10.1128 /$ JB.186.13.4124-4133.2004

Lee, N., and Inouye, M. (1974). Outer membrane proteins of Escherichia coli: biosynthesis and assembly. FEBS Lett. 39, 167-170. doi: 10.1016/00145793(74)80043-8

Lin, J., Cheng, J., Wang, Y., and Shen, X. (2018). The Pseudomonas quinolone signal (PQS): not just for quorum sensing anymore. Front. Cell. Infect. Microbiol. 8:230. doi: 10.3389/fcimb.2018.00230

Magnet, S., Bellais, S., Dubost, L., Fourgeaud, M., Mainardi, J. L., Petit-Frère, S., et al. (2007). Identification of the L,D-transpeptidases responsible for attachment of the Braun lipoprotein to Escherichia coli peptidoglycan. J. Bacteriol. 189, 3927-3931. doi: 10.1128/JB.00084-07

Malinverni, J. C., and Silhavy, T. J. (2009). An ABC transport system that maintains lipid asymmetry in the gram-negative outer membrane. Proc. Natl. Acad. Sci. U. S. A. 106, 8009-8014. doi: 10.1073/pnas.0903229106

Manabe, T., Kato, M., Ueno, T., and Kawasaki, K. (2013). Flagella proteins contribute to the production of outer membrane vesicles from Escherichia coli W3110. Biochem. Biophys. Res. Commun. 441, 151-156. doi: 10.1016/j. bbrc.2013.10.022

Mashburn-Warren, L., Howe, J., Garidel, P., Richter, W., Steiniger, F., Roessle, M., et al. (2008). Interaction of quorum signals with outer membrane lipids: insights into prokaryotic membrane vesicle formation. Mol. Microbiol. 69, 491-502. doi: 10.1111/j.1365-2958.2008.06302.x

Mashburn-Warren, L. M., and Whiteley, M. (2005). Membrane vesicles traffic signals and facilitate group activities in a prokaryote. Nature 437, 422-425. doi: $10.1038 /$ nature 03925

Mashburn-Warren, L. M., and Whiteley, M. (2006). Special delivery: vesicle trafficking in prokaryotes. Mol. Microbiol. 61, 839-846. doi: 10.1111/j. 1365-2958.2006.05272.x

Masilamani, R., Cian, M. B., and Dalebroux, Z. D. (2018). Salmonella Tol-pal teduces outer membrane glycerophospholipid levels for envelope homeostasis and survival during bacteremia. Infect. Immun. 86, e00173-e00218. doi: 10.1128/IAI.00173-18

McBroom, A. J., Johnson, A. P., Vemulapalli, S., and Kuehn, M. J. (2006). Outer membrane vesicle production by Escherichia coli is independent of membrane instability. J. Bacteriol. 188, 5385-5392. doi: 10.1128/JB.00498-06

McBroom, A. J., and Kuehn, M. J. (2007). Release of outer membrane vesicles by gram-negative bacteria is a novel envelope stress response. Mol. Microbiol. 63, 545-558. doi: 10.1111/j.1365-2958.2006.05522.x

Millikan, D. S., and Ruby, E. G. (2004). Vibrio fischeri flagellin a is essential for normal motility and for symbiotic competence during initial squid light organ colonization. J. Bacteriol. 186, 4315-4325. doi: 10.1128/JB.186.13.43154325.2004

Moon, D. C., Choi, C. H., Lee, J. H., Choi, C. W., Kim, H. Y., Park, J. S., et al. (2012). Acinetobacter baumannii outer membrane protein a modulates the biogenesis of outer membrane vesicles. J. Microbiol. 50, 155-160. doi: 10.1007/s12275-012-1589-4.

Murphy, K., Park, A. J., Hao, Y., Brewer, D., Lam, J. S., and Khursigara, C. M. (2014). Influence of $\mathrm{O}$ polysaccharides on biofilm development and outer membrane vesicle biogenesis in Pseudomonas aeruginosa PAO1. J. Bacteriol. 196, 1306-1317. doi: 10.1128/JB.01463-13

Nevermann, J., Silva, A., Otero, C., Oyarzún, D. P., Barrera, B., Gil, F., et al. (2019). Identification of genes involved in biogenesis of outer membrane vesicles (OMVs) in Salmonella enterica serovar Typhi. Front. Microbiol. 10:104. doi: $10.3389 /$ fmicb.2019.00104
Nguyen, T. T., Saxena, A., and Beveridge, T. J. (2003). Effect of surface lipopolysaccharide on the nature of membrane vesicles liberated from the gram-negative bacterium Pseudomonas aeruginosa. J. Electron Microsc. 52, 465-469. doi: 10.1093/jmicro/52.5.465

Nikaido, H. (2003). Molecular basis of bacterial outer membrane permeability revisited. Microbiol. Mol. Biol. Rev. 67, 593-656. doi: 10.1128/mmbr.67.4. 593-656.2003

Ohara, M., Wu, H. C., Sankaran, K., and Rick, P. D. (1999). Identification and characterization of a new lipoprotein, NlpI, in Escherichia coli K-12. J. Bacteriol. 181, 4318-4325. doi: 10.1128/JB.181.14.4318-4325.1999

Paramonov, N. A., Aduse-Opoku, J., Hashim, A., Rangarajan, M., and Curtis, M. A. (2009). Structural analysis of the core region of O-lipopolysaccharide of Porphyromonas gingivalis from mutants defective in $\mathrm{O}$-antigen ligase and O-antigen polymerase. J. Bacteriol. 191, 5272-5282. doi: 10.1128/JB.00019-09

Paramonov, N., Rangarajan, M., Hashim, A., Gallagher, A., Aduse-Opoku, J., Slaney, J. M., et al. (2005). Structural analysis of a novel anionic polysaccharide from Porphyromonas gingivalis strain W50 related to Arg-gingipain glycans. Mol. Microbiol. 58, 847-863. doi: 10.1111/j.1365-2958.2005.04871.x

Pathirana, R. D., and Kaparakis-Liaskos, M. (2016). Bacterial membrane vesicles: biogenesis, immune regulation and pathogenesis. Cell. Microbiol. 18, 1518-1524. doi: $10.1111 / \mathrm{cmi} .12658$

Pazos, M., and Peters, K. (2019). Peptidoglycan. Subcell. Biochem. 92, 127-168. doi: 10.1007/978-3-030-18768-2_5

Perez, J. C., and Groisman, E. A. (2007). Acid pH activation of the PmrA/ PmrB two-component regulatory system of Salmonella enterica. Mol. Microbiol. 63, 283-293. doi: 10.1111/j.1365-2958.2006.05512.x

Raetz, C. R., and Dowhan, W. (1990). Biosynthesis and function of phospholipids in Escherichia coli. J. Biol. Chem. 265, 1235-1238.

Raetz, C. R., and Whitfield, C. (2002). Lipopolysaccharide endotoxins. Annu. Rev. Biochem. 71, 635-700. doi: 10.1146/annurev.biochem.71.110601.135414

Roier, S., Zingl, F. G., Cakar, F., Durakovic, S., Kohl, P., Eichmann, T. O., et al. (2016). A novel mechanism for the biogenesis of outer membrane vesicles in gram-negative bacteria. Nat. Commun. 7:10515. doi: 10.1038/ncomms10515

Rossi, R. M., Yum, L., Agaisse, H., and Payne, S. M. (2017). Cardiolipin synthesis and outer membrane localization are required for Shigella flexneri virulence. MBio 8, e01199-e01217. doi: 10.1128/mBio.01199-17

Rouf, S. F., Ahmad, I., Anwar, N., Vodnala, S. K., Kader, A., Römling, U., et al. (2011). Opposing contributions of polynucleotide phosphorylase and the membrane protein NlpI to biofilm formation by Salmonella enterica serovar Typhimurium. J. Bacteriol. 193, 580-582. doi: 10.1128/JB.00905-10

Sabra, W., Lünsdorf, H., and Zeng, A. P. (2003). Alterations in the formation of lipopolysaccharide and membrane vesicles on the surface of Pseudomonas aeruginosa PAO1 under oxygen stress conditions. Microbiology 149, 2789-2795. doi: $10.1099 /$ mic.0.26443-0

Samsudin, F., Boags, A., Piggot, T. J., and Khalid, S. (2017). Braun's lipoprotein facilitates OmpA interaction with the Escherichia coli cell wall. Biophys. J. 113, 1496-1504. doi: 10.1016/j.bpj.2017.08.011

Schertzer, J. W., and Whiteley, M. (2012). A bilayer-couple model of bacterial outer membrane vesicle biogenesis. MBio 3, e00297-e00311. doi: 10.1128/ mBio.00297-11

Schromm, A. B., Brandenburg, K., Loppnow, H., Moran, A. P., Koch, M. H., Rietschel, E. T., et al. (2000). Biological activities of lipopolysaccharides are determined by the shape of their lipid a portion. Eur. J. Biochem. 267, 2008-2013. doi: 10.1046/j.1432-1327.2000.01204.x

Schwechheimer, C., and Kuehn, M. J. (2013). Synthetic effect between envelope stress and lack of outer membrane vesicle production in Escherichia coli. J. Bacteriol. 195, 4161-4173. doi: 10.1128/JB.02192-12

Schwechheimer, C., Kulp, A., and Kuehn, M. J. (2014). Modulation of bacterial outer membrane vesicle production by envelope structure and content. BMC Microbiol. 14:324. doi: 10.1186/s12866-014-0324-1

Schwechheimer, C., Rodriguez, D. L., and Kuehn, M. J. (2015). NlpI-mediated modulation of outer membrane vesicle production through peptidoglycan dynamics in Escherichia coli. Microbiology 4, 375-389. doi: 10.1002/mbo3.244

Sheetz, M. P., and Singer, S. J. (1974). Biological membranes as bilayer couples. A molecular mechanism of drug-erythrocyte interactions. Proc. Natl. Acad. Sci. U. S. A. 71, 4457-4461. doi: 10.1073/pnas.71.11.4457

Shrivastava, R., and Chng, S. S. (2019). Lipid trafficking across the gramnegative cell envelope. J. Biol. Chem. 294, 14175-14184. doi: 10.1074/jbc. AW119.008139 
Shrivastava, R., Jiang, X., and Chng, S. S. (2017). Outer membrane lipid homeostasis via retrograde phospholipid transport in Escherichia coli. Mol. Microbiol. 106, 395-408. doi: 10.1111/mmi.13772

Silhavy, T. J., Kahne, D., and Walker, S. (2010). The bacterial cell envelope. Cold Spring Harb. Perspect. Biol. 2:a000414. doi: 10.1101/cshperspect.a000414

Sinha, A., Nyongesa, S., Viau, C., Gruenheid, S., Veyrier, F. J., and Moual, H. L. (2019). PmrC (EptA) and CptA negatively affect outer membrane vesicle production in Citrobacter rodentium. J. Bacteriol. 201, e00454-e00518. doi: 10.1128/JB.00454-18

Smith, S. G., Mahon, V., Lambert, M. A., and Fagan, R. (2007). A molecular Swiss army knife: OmpA structure, function and expression. FEMS Microbiol. Lett. 273, 1-11. doi: 10.1111/j.1574-6968.2007.00778.x

Strateva, T., and Mitov, I. (2011). Contribution of an arsenal of virulence factors to pathogenesis of Pseudomonas aeruginosa infections. Ann. Microbiol. 61, 717-732. doi: 10.1007/s13213-011-0273-y

Sutterlin, H. A., Shi, H., May, K. L., Miguel, A., Khare, S., Huang, K. C., et al. (2016). Disruption of lipid homeostasis in the gram-negative cell envelope activates a novel cell death pathway. Proc. Natl. Acad. Sci. U. S. A. 113, E1565-E1574. doi: 10.1073/pnas.1601375113

Suzuki, H., Nishimura, Y., Yasuda, S., Nishimura, A., Yamada, M., and Hirota, Y. (1978). Murein-lipoprotein of Escherichia coli: a protein involved in the stabilization of bacterial cell envelope. Mol. Gen. Genet. 167, 1-9. doi: 10.1007/ BF00270315

Tamayo, R., Choudhury, B., Septer, A., Merighi, M., Carlson, R., and Gunn, J. S. (2005). Identification of cptA, a PmrA-regulated locus required for phosphoethanolamine modification of the Salmonella enterica serovar typhimurium lipopolysaccharide core. J. Bacteriol. 187, 3391-3399. doi: 10.1128/JB.187.10.3391-3399.2005

Tashiro, Y., Inagaki, A., Shimizu, M., Ichikawa, S., Takaya, N., Nakajima-Kambe, T., et al. (2011). Characterization of phospholipids in membrane vesicles derived from Pseudomonas aeruginosa. Biosci. Biotechnol. Biochem. 75, 605-607. doi: 10.1271/bbb.100754

Trent, M. S., Pabich, W., Raetz, C. R., and Miller, S. I. (2001a). A PhoP/ PhoQ-induced lipase (PagL) that catalyzes 3-O-deacylation of lipid a precursors in membranes of Salmonella typhimurium. J. Biol. Chem. 276, 9083-9092. doi: 10.1074/jbc.M010730200

Trent, M. S., Ribeiro, A. A., Lin, S., Cotter, R. J., and Raetz, C. R. (2001b). An inner membrane enzyme in Salmonella and Escherichia coli that transfers 4-amino-4-deoxy-L-arabinose to lipid a: induction on polymyxin-resistant mutants and role of a novel lipid-linked donor. J. Biol. Chem. 276, 43122-43131. doi: 10.1074/jbc.M106961200

Valeru, S. P., Shanan, S., Alossimi, H., Saeed, A., Sandström, G., and Abd, H. (2014). Lack of outer membrane protein a enhances the release of outer membrane vesicles and survival of Vibrio cholerae and suppresses viability of Acanthamoeba castellanii. Int. J. Microbiol. 2014:610190. doi: 10.1155/2014/610190

Valvano, M. A., Messner, P., and Kosma, P. (2002). Novel pathways for biosynthesis of nucleotide-activated glycero-manno-heptose precursors of bacterial glycoproteins and cell surface polysaccharides. Microbiology 148, 1979-1989. doi: 10.1099/00221287-148-7-1979

Weigand, R. A., Vinci, K. D., and Rothfield, L. I. (1976). Morphogenesis of the bacterial division septum: a new class of septation-defective mutants. Proc. Natl. Acad. Sci. U. S. A. 73, 1882-1886. doi: 10.1073/pnas.73.6.1882

Wessel, A. K., Liew, J., Kwon, T., Marcotte, E. M., and Whiteley, M. (2013). Role of Pseudomonas aeruginosa peptidoglycan-associated outer membrane proteins in vesicle formation. J. Bacteriol. 195, 213-219. doi: 10.1128/JB.01253-12

Yamaguchi, K., and Inouye, M. (1988). Lipoprotein 28, an inner membrane protein of Escherichia coli encoded by nlpA, is not essential for growth. J. Bacteriol. 170, 3747-3749. doi: 10.1128/jb.170.8.3747-3749.1988

Yokoyama, F., Kawamoto, J., Imai, T., and Kurihara, T. (2017). Characterization of extracellular membrane vesicles of an Antarctic bacterium, Shewanella livingstonensis Ac10, and their enhanced production by alteration of phospholipid composition. Extremophiles 21, 723-731. doi: 10.1007/ s00792-017-0937-z

Zhou, L., Srisatjaluk, R., Justus, D. E., and Doyle, R. J. (1998). On the origin of membrane vesicles in gram-negative bacteria. FEMS Microbiol. Lett. 163, 223-228. doi: 10.1111/j.1574-6968.1998.tb13049.x

Conflict of Interest: The authors declare that the research was conducted in the absence of any commercial or financial relationships that could be construed as a potential conflict of interest.

Copyright (C) 2021 Avila-Calderón, Ruiz-Palma, Aguilera-Arreola, Velázquez-Guadarrama, Ruiz, Gomez-Lunar, Witonsky and Contreras-Rodríguez. This is an open-access article distributed under the terms of the Creative Commons Attribution License (CC BY). The use, distribution or reproduction in other forums is permitted, provided the original author(s) and the copyright owner(s) are credited and that the original publication in this journal is cited, in accordance with accepted academic practice. No use, distribution or reproduction is permitted which does not comply with these terms. 